\title{
Regionale Wertschöpfung am Beispiel der Regionalinitiativen in Bayern
}

Regionalinitiativen werden oft als Gegenmacht zur Globalisierung gesehen, die deren negative Auswirkungen auf Land und Leute abmindern sollen. Bei der Rückbesinnung auf Wertschöpfung im näheren geografischen Umfeld handelt es sich um gewachsene, etablierte Strukturen. Wie kann das Regionalprinzip eine hohe ökonomische Bedeutung erreichen?

Von Markus Jahl, Christian Kammlott und Dirk Schiereck

\section{Hintergrund}

Die ersten Dekaden des 21. Jahrhunderts sind geprägt von globalen Wertschöpfungsketten, die aber gerade auch vor dem Hintergrund des jüngsten Berichts des Weltklimarates mit Blick auf ihre ökologischen Konsequenzen zunehmend kritisch hinterfragt werden. Dem gegenüber steht das Konzept der regionalen Wertschöpfung (Hahne 1985). Folgt man den Überlegungen von Marsden (2000) und weitergehend Schermer (2010), so entwickelt sich seit den frühen 2000er-Jahren eine neue "Relokalisierung“ gerade im Bereich der Lebensmittelversorgung und des lokalen/regionalen Tourismus, auch als politischer Akt gegen negative Auswirkungen der Globalisierung auf ländliche Regionen. War zuvor meist eine rein individual-ökonomische Argumentation für lokalisierte Produkte der Treiber (Symmank 2019; YouGov 2018), so verbreiterte sich die Perspektive auf regionale Produzenten-Konsumenten-Allianzen zunehmend mit einer politischen Agenda für eine gentechnikfreie, biologische sowie regionale Herstellung (Schermer 2014; Maschkowski et al. 2016).

Dabei finden sich gerade in der Lebensmittelproduktion schon seit einigen Jahrzehnten ökonomische Gegenmodelle zur global integrierten Wertschöpfung, die als Graswurzelbewegungen klassifiziert werden und denen große Bedeutung beim Übergang zu nachhaltigen Produktions- und Konsumsystemen beigemessen wird (Martin et al. 2016). In Deutschland sind heute unter dem Dach des Bundesverbands der Regionalbewegung e. V. (entstanden aus dem Tag der Regionen) 130 Regionalinitiativen verzeichnet, die sich auf regionales Wirtschaften als Beitrag zur erfolgreichen und nachhaltigen Regionalentwicklung und für eine Stärkung ländlicher Räume konzentrieren (Sindel 2019). Diese recht unspezifische, un- scharfe Umschreibung erlaubt einen erheblichen Interpretationsspielraum und trägt dazu bei, dass es kaum ein klares Bild von Größe, Organisationsstrukturen und Ausrichtung der Regionalinitiativen in Deutschland gibt, und eine ökonomische wie auch nachhaltigkeitsorientierte Bewertung dieser Institutionen in der breiten Öffentlichkeit bislang unterbleibt. Hier setzt dieser Beitrag an, der die Gruppe der bayerischen Regionalinitiativen, die ein Drittel der insgesamt in Deutschland im Verband Regionalbewegung organisierten Initiativen umfasst, im Rahmen einer beispielhaften Illustration vorstellt, insbesondere die Heterogenität innerhalb der Gruppe erläutert und ein Konzept zur Quantifizierung und Erhöhung der Transparenz im Bereich der regionalen Wertschöpfung skizziert.

\section{Die heterogene Struktur der Regional- initiativen am Beispiel Bayerns}

Jenseits der individuell ausgerichteten regionalen Wertschöpfung eines jeden einzelnen Unternehmens wird gleichgerichtetes regional orientiertes Wirtschaften auf unternehmensübergreifender Ebene in Deutschland maßgeblich durch Regionalinitiativen organisiert, von denen $130 \mathrm{im}$ Verband der Regionalbewegung e. V. vertreten sind. Ein Drittel dieser Vereinsmitglieder hat seinen wirtschaftlichen Aktivitätsfokus im Freistaat Bayern. Diese Gruppe wird nachfolgend hinsichtlich ihrer wesentlichen Charakteristika institutionell analysiert.

Insgesamt konnten zur weiteren Analyse Daten für 44 Regionalinitiativen aus Bayern zusammengetragen werden. Alle entstammen dem Netzwerk Regionalbewegung e. V. und bestehen zum Teil schon mehrere Jahrzehnte. Erste, heute noch aktive Gründungen gab es bereits in den Jahren 1963 und 1984. Während 2004 das Jahr mit den meisten Neugründungen (6) war, kamen nach 2008 trotz ökologischem Boom - gerade auch in der Landwirtschaft - insgesamt nur noch zehn neu gegründete Initiativen hinzu. Diese Gründungsdaten unterstreichen, dass die Idee der Regionalinitiativen keinem aktuellen modischen Trend entstammt, sondern auf lang etablierten Säulen ruht. Trotz ihres schon fortgeschrittenen Alters bleiben die Regionalinitiativen ihrem geografischen Fokus treu und in der Mitgliederzahl dadurch auch überschaubar groß. Die Mehrzahl der Initiativen (28) hat weniger als 100 Mitglieder, wobei Mitglieder hier als teilnehmende Betriebe, Mitglieder im Verein beziehungsweise der Firmierung der Initiative erfasst werden. Immerhin 10 Initiativen zählen 100 bis 500 Mitglieder, und 6 haben zwischen 500 und 1.000 Mitglieder. Die größten Initiativen sind die Allgäu GmbH mit 1.100 Mitgliedern und die Re- 


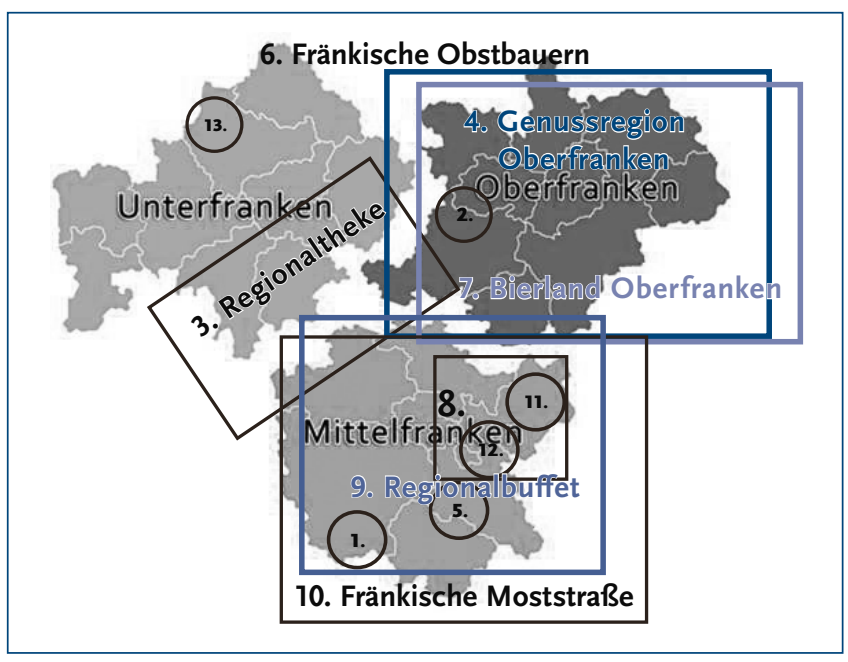

Abbildung 1: Überschneidungen der Regionalinitiativen in Franken

gion Hesselberger mit fast 2.000 zuliefernden Obstbauern. Eine Erfassung der Mitgliederzahlen im Zeitablauf und damit eine Indikation der fortschreitenden Durchdringung der jeweiligen Region war datenseitig leider nicht durchgängig möglich.

Mit der Variation in der Größe gehen auch Unterschiede in der Rechtsformwahl einher. Die Mehrheit der bayerischen Regionalinitiativen firmiert unter einem gemeinnützigen Verein (26). Neben den acht GmbHs drückt sich ein oft hohes Demokratieverständnis der Initiativen mit der Firmierung bei zwei Genossenschaften, zwei Interessengemeinschaften und einer GbR aus. Öffentlich-rechtliche Rahmenstrukturen finden sich bei vier Initiativen, die direkt vom Landratsamt oder Landkreis gesteuert sind.

Schon diese ersten Daten zeigen sehr klar, dass bereits innerhalb der Gruppe bayerischer Regionalinitiativen eine große Heterogenität herrscht und es die typische Regionalinitiative nicht gibt, was für Graswurzelbewegungen auch eher typisch als überraschend ist. Diese Einsicht wird weiter unterstrichen, wenn die regionale Ausdehnung der einzelnen Initiativen betrachtet wird. Engflächig bis $100 \mathrm{~km}^{2}$ arbeiten nur fünf Regionalinitiativen, bis $500 \mathrm{~km}^{2}$ beziehungsweise bis $1.000 \mathrm{~km}^{2}$ jeweils sechs, und darüber hinaus mit einer Ausdehnung bis $5.000 \mathrm{~km}^{2}$ sind 16 Initiativen aktiv. Größer als $5.000 \mathrm{~km}^{2}$ ist die regionale Ausdehnung bei elf Initiativen. Die Bezeichnung der „Regionalinitiative“ erscheint also unbedingt angemessen, die Zusammenschlüsse überschreiten die lokale Ebene in der Regel deutlich. Einschränkend ist dabei allerdings eine gewisse Rivalität unter den Regionalinitiativen zu beachten, viele Initiativen überlappen sich in ihrem Aktionsgebiet oder beanspruchen gar das gleiche unter variierender Ausrichtung.

Zur Illustration dieser geografischen Überschneidungen in den Aktionsgebieten verschiedener Regionalinitiativen hilft ein Blick auf Franken mit seinen Unterregionen Unter-, Mittelund Oberfranken (Abbildung 1).

In Franken sind insgesamt zwölf Regionalinitiativen aktiv und zwar alle im Bereich der Lebensmittelvermarktung. Die in Abbildung 1 eingezeichneten Rechtecke und Kreise deuten die eher uneindeutig festgelegten Gebietseingrenzungen der Initiativen an. Nur in Unterfranken gibt es keine Überschneidungen, so agieren die Regionaltheke (3.) in Teilen Unterfrankens und Mittelfrankens und die Initiative Schlaraffenburger (13.) im Landkreis Aschaffenburg. Überregional in ganz Franken ist die Initiative Fränkische Obstbauern (6.) tätig. Diese Fläche wird aber auch von der Initiative Schlaraffenburger in Teilen vermarktet, ebenso wie von den Initiativen Streuobst Hesselberg (1.), Brombachseer Kirschen (5.), Metropolregion Nürnberg (12.), Kalchreuther Kirschen (11.) und Heimat auf'm Teller (8.). Die Aktionsgebiete dieser zuletzt genannten Initiativen werden wiederum von den Initiativen Regionalbuffet (9.) und Fränkische Moststraße (10.) überlagert. In Oberfranken überschneiden sich zudem das Bierland Oberfranken (7.) und die Genussregion Oberfranken (4.). Diese Strukturen unterstreichen auch, dass manche Initiativen ihre Einzigartigkeit nicht aus historischen Definitionen von Regionen ableiten, sondern dass die gewählten regionalen Abgrenzungen ökonomischen Kalkülen folgen.

Generell wird zwischen einer analytischen und synthetischen geografischen Regionsabgrenzung unterschieden. Bei der analytischen Unterscheidung ist der maßgebendste Faktor die Abgrenzung, bei der synthetischen die größtmögliche Anzahl an Merkmalen. Bei 34 Initiativen wurde nach dem maßgebendsten Faktor (Beispiel: Ammergauer Alpen - Ammergau als Merkmal) und bei 10 nach der Anzahl an Merkmalen (Beispiel: Fränkische Moststraße - Wein, Frankenland, teilnehmender Mostbetrieb [...] als Merkmal) abgegrenzt. Eine weitere Unterteilung kann nach dem Homogenitäts-, Funktional- und Verwaltungsprinzip erfolgen. Das Homogenitätsprinzip sieht eine Abgrenzung durch ähnliche Struktur vor (Pro-Kopf-Einkommen von fränkischen Bauern, Beispiel: Die Regionaltheke), das Funktionalprinzip durch interne Verflechtungen (Mehrzahl der Arbeitsplätze, Beispiel: Direktvermarkter zwischen Watzmann und Waginger See) und das Verwaltungsprinzip durch Gemeinde oder Stadt. Von den 44 Initiativen im Vergleich grenzen sich 18 durch das Homogenitätsprinzip, 5 nach dem Funktionalprinzip und 21 nach dem Verwaltungsprinzip ab. Somit kann es durchaus sein, dass sich eine Stadt oder Gemeinde hinter eine Regionalinitiative stellt, aber auch, dass eine Gemeinschaft von Gleichgesinnten sich etwas Gemeinsames schafft.

Die Überlappungen in den geografischen Einzugsgebieten der Regionalinitiativen werden oft bereits durch den Produktfokus und die Notwendigkeit einer gewissen Mindestgröße getrieben, wie aus der Namensgebung der oben erwähnten Initiativen zum Teil schon deutlich wird. Während die meisten Initiativen ihren Fokus allgemein auf Lebensmittel als Sammelkategorie legen, versuchen einige andere auf spezifischere Produktgruppen als Alleinstellungsmerkmale abzuzielen. Neben einem breiten Fokus auf regionale Lebensmittel gibt es Konzentrationen auf den Gemüse- und Kräuteranbau, auf Eier sowie Milch und Molkereiprodukte. Zudem gibt es Ausrichtungen auf den Tourismus und auf Streuobstinitiativen, auf den 
Erhalt lokaler und regionaler Spezialitäten in der Küche konzentrieren sich vier Initiativen.

\section{Regionalität, regionale Wertschöpfung und das Problem der Quantifizierung}

Wie eingangs erwähnt, verfolgen Regionalinitiativen neben einer rein individual-ökonomischen Argumentation für lokalisierte Produkte auch zunehmend eine politische Agenda. So streichen 18 Initiativen das reduzierte Verkehrsaufkommen durch lokale Transporte als positiven gesamtgesellschaftlichen Nutzen und Wert von Regionalität heraus. 28 Initiativen sehen durch ihr Werk die Landschaft und die kulturelle Vielfalt geschützt, 19 Initiativen werben mit mehr Lebensmittelsicherheit durch kleinere Kreisläufe, und 18 loben die Verbesserung der Produktqualität durch die Nähe zum Erzeuger.

Regionalinitiativen schützen und sichern mit ihrer Arbeit dezentrale Arbeitsplätze, acht betonen explizit die Erhöhung der regionalen Wertschöpfung durch ihr Handeln. Die regionale Wertschöpfung hat sich als Vermarktungsargument offensichtlich zumindest so gut etabliert, dass diese inzwischen zu den Top-3-Kriterien bei der Lebensmittelauswahl in Deutschland gilt (YouGov 2018) und verschiedene große Supermarktketten wie REWE oder Edeka für ihr Produktsortiment darauf besonders verweisen. Die zunehmende Verwendung des Begriffs zeigt aber auch die drängende Notwendigkeit der begrifflichen Spezifikation und damit der Quantifizierung. Wenn nicht jedes Unternehmen und jeder Unternehmensverbund für sich in Anspruch nehmen dürfen soll, Produkte aus ganz überwiegend regionaler Wertschöpfung anzubieten, gilt es, Messkonzepte für diese Wertschöpfung zu entwickeln. Eine einheitliche und etablierte Methode dazu existiert bislang nicht. Die meisten vorliegenden Konzepte fokussieren insgesamt bestimmte Regionen, ohne dabei tiefergehend auf die individuelle Situation beziehungsweise den Beitrag einzelner Akteure einzugehen. Aber es sind ja gerade die jeweiligen Unternehmen als die originären Träger der Wertschöpfung, die ihre Entscheidungen und strategischen Überlegungen am Konzept der regionalen Wertschöpfung ausrichten müssen, um ihren regionalen Wertschöpfungsbeitrag messen und optimieren zu können. Daraus ergeben sich die folgenden Anforderungen für ein adäquates Messkonzept:

- Fokussierung auf die Akteure,

I hoher Objektivierungsgrad mit einem Algorithmus, der einer einheitlichen und objektiven Systematik folgt,

I einfache Interpretierbarkeit.

Erste Überlegungen und Anwendungsbeispiele für ein solches Konzept erläutern exemplarisch Kammlott und Schiereck (2018) mit ihrem Modell zur Quantifizierung regionaler Wertschöpfungsbeiträge auf der Basis von Daten aus dem Rechnungswesen der jeweiligen Unternehmen. Dabei wurde im Rahmen des Projekts Bio \& Faire Wertschöpfungsketten in Regionen in enger Zusammenarbeit mit dem BioFairVerein und gefördert durch das Bundesprogramm ökologischer Landbau und andere Formen nachhaltiger Landwirtschaft (BÖLN) ein erster Schritt zur Entwicklung eines solchen Bewertungsverfahrens entwickelt. Die Kalkulation basiert auf internen Rechnungslegungsdaten der mitwirkenden Unternehmen, bedarf also der aktiven Mitarbeit des zu bewertenden Unternehmens. So werden sämtliche Buchungsvorgänge eines Jahrganges in einen Regionalbezug gesetzt und damit der Wertschöpfungsbeitrag des Unternehmens für das Bezugsjahr absolut, in Euro-Beträgen, sowie relativ, im Verhältnis zu den Umsatzerlösen, ermittelt. Darüber hinaus wird unter Anwendung der im Bereich Unternehmensbewertung etablierten finanzmathematischen Verfahren ein sogenannter "Regionaler Unternehmenswert" berechnet, der den aggregierten langfristigen Wertbeitrag des Unternehmens für eine Region ausdrückt. Dieses neue Verfahren hat den großen Vorteil, dass mehrere Unternehmen direkt miteinander verglichen und so deren Wertschöpfungsbeiträge innerhalb einer Gruppe von Unternehmen evaluiert und in eine Ordnung gebracht werden können.

Soziale und ökologische Faktoren, die beispielsweise aus der Verringerung der räumlichen Distanz zwischen Erzeuger/innen und Verbraucher/innen resultieren können, messen solche Modelle indes noch nicht. Dass es hierbei relevante Unterschiede gibt, wurde auch bei der Untersuchung der bayerischen Regionalinitiativen deutlich, bei denen der Einklang von regionaler Wertschöpfung und regionaler Vermarktung sehr unterschiedlich ausgeprägt ist. So nutzen 31 Regionalinitiativen den Verkauf unmittelbar beim Produzenten und sind so in der Lage, eine unmittelbare Beziehung zum Verbraucher zu etablieren. 24 vertreiben ihre Produkte nur innerhalb ihrer Region und vier Initiativen vermarkten diese auch überregional mit Herkunftsverweisen, wobei Mehrfachklassifikationen möglich waren. Die Heterogenität der Vertriebsstrategien unterstreicht, dass Regionalität im Lebensmittelkonsum stark von lokalen funktionierenden Infrastrukturen abhängt (Maschkowski et al. 2016).

\section{Fazit}

Als Gegenpol zu aktuellen Megatrends wie Globalisierung und Urbanisierung, die neben mannigfaltigen wirtschaftlichen Vorteilen eine Vielzahl von ökologischen und ökonomischen Nachteilen auch für ländlich geprägte Räume mit sich bringen, hat sich in den vergangenen Jahrzehnten eine Rückbesinnung auf lokale, regional geprägte Wertschöpfungsstrukturen entwickelt, die sich unter anderem in der Etablierung einer Vielzahl von Regionalinitiativen manifestiert hat. Den Konsument/innen scheint dieser Trend grundsätzlich zu gefallen. Das wird unmittelbar deutlich, wenn man sich aktuelle Slogans wie „von hier“, „Ein gutes Stück Heimat" oder „Unsere Heimat - echt\& gut" ins Gedächtnis ruft, deren sich inzwischen nahezu der gesamte Einzelhandel bedient. Die Untersuchungen von Hausruckinger und Helm (1996) und von Alvensleben (2000) bestätigen eine emotionale Qualität, insofern die Heimatregion emotional positiv belegt ist. Dies führt 
dazu, dass man bei regionalen Produkten an etwas Gutes, Heimisches denkt (YouGov 2018). Offen bleibt dabei aber fast immer eine klare und aussagekräftige Definition solcher Begriffe, denn viele Konsument/innen scheinen den nächsten Schritt, die Überprüfung dieser positiven Gedanken, noch nicht zu gehen, was wiederum zu einer Inflationierung der Idee führen und die dauerhafte Akzeptanz von Regionalität und regionaler Wertschöpfung als positive Verbindung ökonomischer und ökologischer Komponenten unterminieren kann.

Regionalinitiativen würde im internationalen Kontext die Bezeichnung der grassroots initiatives zufallen, denen beispielsweise Grabs et al. (2016) nicht nur eine erhebliche Bedeutung beimessen, um mit Verhaltensmodellen, die über das individuelle Wohlbefinden und den Materialverbrauch hinausgehen, nachhaltige Gesellschaften zu erreichen. Vielmehr sehen sie in entsprechenden Bewegungen einen elementaren Grundbaustein, mit dem die sozialen Beziehungen, die Übernahme von Verantwortlichkeiten für andere Menschen und Nicht-Menschen sowie das bürgerschaftliche Engagement als ergänzende Quellen des Wohlbefindens berücksichtigt werden, um die individuellen Fußabdrücke zu verringern (Martin et al. 2016). Entsprechend sehen Grab et al. (2016) Basisinitiativen, die kollektives Handeln und soziales Lernen anregen, als Vorbilder für den gesellschaftlichen Wandel.

Am Beispiel der Regionalinitiativen in Bayern wird sehr schnell deutlich, wie wenig homogen diese Organisationen sind. Es lässt sich eine breite Diversität im Hinblick auf Größe, Ausdehnungsgebiet und inhaltliche Orientierung beobachten. Das ist auch nicht überraschend vor dem Hintergrund, dass es keinerlei Regulierung oder einheitliche Systematik und Definitionskriterien gibt. Gerade die begriffliche Klarheit und Überprüfbarkeit würde aber nicht nur mehr Vertrauen und Glaubwürdigkeit in dieses Segment bringen, sondern auch den Trägern dieser Initiativen, den mittelständischen Familienbetrieben und regionalen Akteuren, womöglich gute Argumente liefern, die gegenüber den Verbrauchern als Marketinginstrument genutzt werden könnten.

Daneben bieten eine einheitliche Klassifizierung und die Herleitung von Erfolgsmerkmalen wie zum Beispiel die Quantifizierung regionaler Wertschöpfungsbeiträge wichtige Argumentationsgrundlagen für die Abstimmung mit öffentlichen Einrichtungen, den Gemeinden und Regionalverwaltungen bis hin zu Fördereinrichtungen. Nicht zuletzt ließe sich damit auch das eigene Wertverständnis erheblich besser messen und steuern. Der wirkliche Wertbeitrag solcher Initiativen würde dann womöglich transparenter sowie besser kommuniziert und verstanden werden, und es resultierte eine deutlich klarere Abgrenzung gegenüber einfachen Marketingaussagen, die heute gleichsam auf Augenhöhe neben tatsächlich wertschaffenden Regionalinitiativen koexistieren. Und so müssen sich viele Regionalinitiativen bis heute die Kritik gefallen lassen, dass sie nicht genau wissen (oder wissen können), welche Auswirkungen ihr Handeln quantitativ für (die nicht immer vollkommen klar definierte) Region hat.

\section{Literatur}

Alvensleben, R. von (2000): Zur Bedeutung von Emotionen bei der Bildung von Präferenzen für nationale Produkte. In: Agrarwirtschaft 49/12: 399-402.

Grabs, J./Langen, N./Maschkowski, G./Schäpke, N. (2016): Understanding Role Models for Change: A Multilevel Analysis of Successfactors of Grassroots Initiatives for Sustainable Consumption. In: Journal of Cleaner Production 134/2: 98-111.

Hahne, U. (1985): Regionalentwicklung durch Aktivierung intraregionaler Potentiale: Zu den Chancen „endogener“ Entwicklungsstrategien. München, Universität Kiel.

Hausruckinger, G./Helm, R. (1996): Die Bedeutung des Country-of-Origin Effekts vor dem Hintergrund der Internationalisierung von Unternehmen: Eine teilweise individualisierte Conjoint-Analyse. In: ZFP - Journal of Research and Management 18/4: 267-278.

Kammlott, C./Schiereck, D. (2018): Zur Qualifizierung regionaler Wertschöpfungsbeiträge: Regionale Wertschöpfung als Forschungsgegenstand. In: Teitscheid, P./Langen, N./Speck, M./Rohn, H. (Hrsg.): Nachhaltig außer Haus essen. München, oekom. 140-152.

Marsden, T./Banks, J./Bristow, G. (2000): Food Supply Chain Approaches: Exploring their Role in Rural Development. In: Sociologia Ruralis 40/4: 425-439.

Martin, C. J./Upham, P. (2016): Grassroots Social Innovation and the Mobilisation of Values in Collaborative Consumption: A Conceptual Model. In: Journal of Cleaner Production 134/2: 204-213.

Maschkowski, G./Klein, B. (2016): Regional einkaufen. Praxistest zur Verbesserung der Ernährungskommunikation. In: Ernährung im Fokus 16/7-8: 228-231.

Schermer, M. (2015): From "Food from Nowhere" to "Food from Here:" Changing Producer-consumer Relations in Austria. In: Agriculture and Human Values 32/1: 121-132.

Schermer, M. (2010): Oft mehr Wertschätzung als Wertschöpfung. In: Raum 2010/80: 25-26.

Sindel, H. (2019): Regionalverband e. V., Die Idee - Dachverband und Interessenvertretung für Regionalinitiativen. Internet: https://www.regionalbewegung.de/bewegung/idee/

Symmank, C. (2019): Extrinsic and Intrinsic Food Product Attributes in Consumer and Sensory Research: Literature Review and Quantification of Findings. In: Management Review Quarterly 69/1: 39-74.

YouGov (Hrsg.) (2018): Regionalität als Kaufkriterium bei Lebensmitteln. Köln, YouGov.

\section{AUTOREN + KONTAKT}

Markus Jahl ist Human Resources Specialist bei der Atomic Austria $\mathrm{GmbH}$.

Atomic Austria GmbH, Atomic Straße 1, 5541 Altenmarkt im Pongau. Tel.: +436452 3900221, E-Mail: markus_jahl@me.com,

Website: https://www.atomic.com/de-at

Dr. Christian Kammlott ist Professor für allgemeine Betriebswirtschaft, insbesondere Corporate Finance und Entrepreneurship an der Hochschule Trier. Hochschule Trier, Umwelt-Campus Birkenfeld, Campusallee, 55768 Hoppstädten-Weiersbach. Tel.: +49 6782171117,

E-Mail: c.kammlott@umwelt-campus.de https://www.umwelt-campus.de/

Dr. Dirk Schiereck ist Professor für Unternehmensfinanzierung und Leiter des Fachgebiets Corporate Finance an der Technischen Universität Darmstadt.

Technische Universität Darmstadt, Hochschulstraße 1, 64289 Darmstadt. Tel.: +496151 1624291, E-Mail: schiereck@bwl.tu-darmstadt.de, Website: https://www.tu-darmstadt.de/

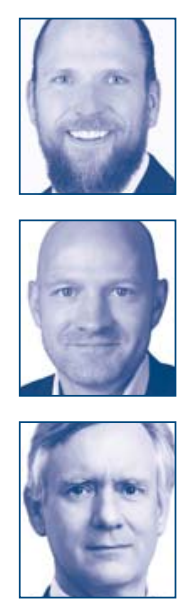

